\title{
Constraining the mass and moment of inertia of neutron star from quasi-periodic oscillations in $\mathrm{X}$-ray binaries
}

\author{
Jérôme Pétri* \\ Observatoire Astronomique de Strasbourg, UMR 7550 Université de Strasbourg, CNRS, 11 rue \\ de l'Université, 67000 Strasbourg, France \\ E-mail: jerome.petri@astro.unistra.fr
}

\begin{abstract}
Neutron stars are the densest objects known in the Universe. Being the final product of stellar evolution, their internal composition and structure is rather poorly constrained by measurements. We put some constraints on the mass and moment of inertia of these neutron stars based on the interpretation of quasi-periodic oscillations observed in low mass X-ray binaries (LMXBs). More specifically, we use observations of high-frequency quasi-periodic observations (HF-QPOs) to look for the average mass and moment of inertia of neutron stars. This is done by applying our parametric resonance model to discriminate between slow and fast rotators. We fit our model to data from ten LMXBs for which HF-QPOs have been seen and the spin of the enclosed accreting neutron star is known. We find an average mass $M_{*} \approx 2.0-2.2 M_{\odot}$. The corresponding average moment of inertia is then $I_{*} \approx 1-3 \times 10^{38} \mathrm{~kg} \mathrm{~m}^{2} \approx 0.5-1.5(10 \mathrm{~km})^{2} M_{\odot}$.
\end{abstract}

Fast $X$-ray timing and spectroscopy at extreme count rates

February 7-11, 2011

Champéry, Switzerland

* Speaker. 


\section{Introduction}

Neutron stars are excellent astrophysical laboratories to test matter above nuclear density [1]. Unfortunately, there is nowadays no way for nuclear physicists to investigate matter at such extremely high densities. Moreover, because of the lack of knowledge about the behavior of particles in these extreme regimes, there is yet no consensus on a satisfactory equation of state for nucleons in those compact objects although many equations of state being proposed. Measuring the mass $M$ and the radius $R$ of these neutron stars should help to constrain the equations of state and possibly reject some of them [2].

HF-QPOs observations in LMXBs is a unique tool to test gravity in the strong field regime and to learn about the behavior of particles at high densities. For these accreting neutron stars, some attempts have been undertaken to constrain $M$ and $R$. Because during their life neutron stars in binaries accrete matter from their companion, infalling matter can reach a substantial fraction of their initial mass. Thus, not surprisingly, we will expect final masses deviating significantly from the fiducial $1.4 M_{\odot}$.

Therefore, quasi-periodic oscillations can help to diagnose motion in strong gravitational fields and maybe solve the problem of determining $M$ and $R$. In this work, we show how to estimate the average mass and moment of inertia of neutron stars by fitting kHz-QPO observations in LMXBs for slow and fast rotators.

\section{Model and method}

The essential feature of the model is the presence of a rotating neutron star which possesses an asymmetry about its rotation axis. [3] has shown that this induces some driven motion in the accretion disk due to a parametric resonance. Therefore the disk will show strong response to this drive by oscillating across the equatorial plane at some given preferred radii where the resonance condition is satisfied.

Vertical resonance occurs whenever the vertical epicyclic frequency is equal to the perturbation frequency as measured in the locally corotating frame such that

$$
m\left|\Omega\left(r, a_{*}\right)-\Omega_{*}\right|=2 \frac{\kappa_{\mathrm{z}}\left(r, a_{*}\right)}{n}
$$

where $m$ is the azimuthal number of the perturbation mode, $\Omega\left(r, a_{*}\right)$ the orbital frequency in the disk at radius $r, \Omega_{*}$ the spin of the neutron star, $a_{*}$ a length related to the angular momentum $J_{*}=I_{*} \Omega_{*}$ by $a_{*}=J_{*} / M_{*} c, \kappa_{\mathrm{z}}\left(r, a_{*}\right)$ the vertical epicyclic frequency, $I_{*}$ the stellar moment of inertia and $n$ an integer. The frequencies $\Omega$ and $\kappa_{\mathrm{z}}$ are expressed for a test particle in Kerr space-time. They depend explicitly on the radius $r$ and on the angular momentum $a_{*}$ as

$$
\begin{aligned}
& \Omega\left(r, a_{*}\right)=\frac{\sqrt{G M_{*}}}{r^{3 / 2}+a_{*} \sqrt{R_{\mathrm{g}}}}=\frac{c^{3}}{G M_{*}} \frac{1}{\tilde{r}^{3 / 2}+\tilde{a}} \\
& \kappa_{\mathrm{z}}\left(r, a_{*}\right)=\Omega\left(r, a_{*}\right) \sqrt{1-4 \frac{\tilde{a}}{\tilde{r}^{3 / 2}}+3 \frac{\tilde{a}^{2}}{\tilde{r}^{2}}}
\end{aligned}
$$

$R_{\mathrm{g}}=G M_{*} / c^{2}$ is the gravitational radius of the star, $\tilde{r}=r / R_{\mathrm{g}}$ and $\tilde{a}=a_{*} / R_{\mathrm{g}}$. 
From the known spin of the neutron star, we can deduce its angular moment by $J_{*}=I_{*} \Omega_{*}$, assuming a given value for the moment of inertia $I_{*}$. Therefore, guessing a mass and a moment of inertia, we can solve quantitatively Eq. (2.1) for the orbital frequency $\Omega$ and try to match observations of kHz-QPOs. In the general case, for arbitrary $\tilde{a}$, the resonance condition Eq. (2.1) must be solved numerically. However, for slowly rotating stars, $\tilde{a} \ll 1$, we retrieve the Newtonian expression $\Omega\left(r, a_{*}\right) \approx \Omega(r, 0)=\kappa_{\mathrm{z}}(r, 0)=\sqrt{\frac{G M_{*}}{r^{3 / 2}}}$ from which the solution of Eq. (2.1) follows immediately

$$
\Omega(r, 0)=\frac{m n}{m n \pm 2} \Omega_{*}
$$

The orbital frequency $\Omega(r, 0)$ should remain smaller than this at the innermost stable circular orbit (ISCO) given in the non-rotating limit by

$$
v_{\text {isco }}=2198 \mathrm{~Hz}\left(\frac{M_{\odot}}{M_{*}}\right)=1570 \mathrm{~Hz}\left(\frac{M_{*}}{1.4 M_{\odot}}\right)^{-1}
$$

This would give a first guess for the expected QPO frequencies, knowing the mass $M_{*}$. Actually, because the spin frequency is well known from X-ray bursts for instance, we can do better and include the angular momentum $\tilde{a}$ into the description, but then the moment of inertia comes in as another free parameter.

Several LMXBs have been observed with known spin rate and showing the twin peak QPO phenomenon, see for instance [4]. Depending on the neutron star rotation speed, they have been classified as slow rotator for $\nu_{*} \lesssim 400 \mathrm{~Hz}$ or as fast rotator for $v_{*} \gtrsim 400 \mathrm{~Hz},\left(v_{*}=\Omega_{*} / 2 \pi\right)$. For slow rotators, the twin $\mathrm{kHz}-\mathrm{QPO}$ difference is almost equal to the spin frequency, $\Delta v^{\mathrm{obs}} \approx v_{*}$, while for fast rotators, it is equal to half of it, $\Delta v^{\mathrm{obs}} \approx v_{*} / 2$. In our model, this is interpreted as an indirect consequence of the ISCO. We also include a neutron star spin-dependent mass based on some heuristic argument [5]. The rotation of the neutron star, containing a fixed number of $N$ nucleons, increases its gravitational mass $M\left(N, \Omega_{*} \neq 0\right)$ compared to the non rotating limit $M\left(N, \Omega_{*}=0\right)$ due to its rotational kinetic energy. We find a relative mass correction of

$$
\frac{\delta M}{M_{\odot}}=\frac{I_{*} \Omega_{*}^{2}}{2 c^{2} M_{\odot}}=1.76 \times 10^{-3}\left(\frac{I_{*}}{10^{38} \mathrm{~kg} \mathrm{~m}^{2}}\right)\left(\frac{v_{*}}{400 \mathrm{~Hz}}\right)^{2}
$$

So it remains small even for fast rotators.

\section{Results}

The above model is applied to a handful of fast and slow rotators. We emphasize that in order to make any prediction on the mass and moment of inertia, we have to take the same properties for the whole sample of accreting neutron stars in the observed LMXBs. Indeed, adopting different parameters for each system could significantly change the orbital and epicyclic frequencies. Most importantly, the frequency at the ISCO, scaling like $1 / M_{*}$ would change from one binary to another. But in our segregation between slow and fast rotators, the precise value of the orbital frequency at the ISCO is the salient feature to interpret the abrupt change in the twin peak frequency difference. A varying $M_{*}$ would shift this sharp transition to lower or higher frequencies from one binary to another. Thus the zero-th order choice, to highlight the general trend, is to keep the same mass for all neutron stars. 
Let us give an estimate for the gravitational mass $M_{*}$ and moment of inertia $I_{*}$ along the following arguments. The geometrized spin parameter $\tilde{a}$ is defined as

$$
\tilde{a}=\frac{I_{*} \Omega_{*} c}{G M_{*}^{2}}=0.145\left(\frac{I_{*}}{10^{38} \mathrm{~kg} \mathrm{~m}^{2}}\right)\left(\frac{v_{*}}{400 \mathrm{~Hz}}\right)\left(\frac{M_{*}}{1.4 M_{\odot}}\right)^{-2}
$$

From this expression it is clear that it remains small compared to unity, and this even for fast rotators. In this case, to first order in $\tilde{a}$, the orbital frequency at the ISCO is [6]

$$
v_{\text {isco }}(\tilde{a})=2198 \mathrm{~Hz}(1+0.75 \tilde{a}) \frac{M_{\odot}}{M_{*}}
$$

Assuming that $\tilde{a} \leq 0.3$, an inaccuracy introduced by this simplification with respect to the Kerr solution is smaller than $10 \%$. We put explicitly the spin dependence through $\tilde{a}$ on the left hand side for latter convenience. And therefore the relation between $M_{*}$ and $I_{*}$ becomes approximately

$$
I_{*}=\frac{2}{3 \pi} \frac{G M_{*}^{2}}{c v_{*}}\left[\left(\frac{v_{\mathrm{isco}}(\tilde{a})}{2198 \mathrm{~Hz}}\right) \frac{M_{*}}{M_{\odot}}-1\right]
$$

According to our parametric resonance model, for slowly rotating stars, the twin kHz-QPOs are given by $v_{1}^{\mathrm{s}}=2 v_{*}$ and $v_{2}^{\mathrm{s}}=3 v_{*}$, where the superscript s stands for slow. Because $\left(v_{1}^{\mathrm{s}}, v_{2}^{\mathrm{s}}\right)$ are interpreted as the frequencies of the orbital motion, they need to be less than that at the ISCO

$$
\left(v_{1}^{\mathrm{s}}, v_{2}^{\mathrm{s}}\right) \leq v_{\text {isco }}
$$

For increasing spin of the neutron star $v_{*}$, at some point, $v_{2}^{\mathrm{s}}$ will approach and eventually overtake $v_{\text {isco. Thus }} v_{2}^{\mathrm{s}}$ will be forbidden as a HF-QPO. As a consequence, the next two dominant twin kHz-QPOs are identified as $v_{1}^{\mathrm{f}}=1.5 v_{*}$ and $v_{2}^{\mathrm{f}}=2 v_{*}$. Therefore, the QPO frequency difference $\Delta v / v_{*}=\left(v_{2}-v_{1}\right) / \nu_{*}$ jumps suddenly from 1.0 and 0.5 . According to the data taken from [7], this should happen in the neutron star spin range $v_{*} \in[363,401] \mathrm{Hz}$. This is probably the most salient feature in the slow against fast rotator discrepancies. Fitting these data requires that the switching from slow to fast rotator occurs for neutron star spin between $363 \mathrm{~Hz}$ and $401 \mathrm{~Hz}$. More precisely, for $v_{*} \leq 363 \mathrm{~Hz}, \Delta v / v_{*} \approx 1$ which we interpret as no effect on motion in the observable disk from the presence of an ISCO. This implies that $v_{\text {isco }}(363 \mathrm{~Hz}) \geq 3 v_{*}=1089 \mathrm{~Hz}$, we put the spin rate into coma to distinguish between different rotators, an essential remark for our constraints. Next, for $v_{*} \geq 401 \mathrm{~Hz}, \Delta v / \nu_{*} \approx 0.5$ which we interpret as a clear signature of the ISCO. This implies that $v_{\text {isco }}(401 \mathrm{~Hz}) \leq 3 v *=1203 \mathrm{~Hz}$. Express in terms of the ISCO, the transition from slow to fast rotator should happen when the two conditions below are satisfied

$$
\begin{aligned}
& v_{\text {isco }}(363 \mathrm{~Hz}) \geq 1089 \mathrm{~Hz} \\
& v_{\text {isco }}(401 \mathrm{~Hz}) \leq 1203 \mathrm{~Hz} .
\end{aligned}
$$

This condition supplemented with the relation Eq. (3.3) sets two constraints on $M_{*}$ and $I_{*}$, an allowed region in the $\left(M_{*}, I_{*}\right)$ plane. Next, a third bound for the couple $\left(M_{*}, I_{*}\right)$ is possible along the following lines. For fast rotators, the ISCO is clearly taken into account. But for the highest accreting system with $v_{*}=619 \mathrm{~Hz}$, the ratio is still $\Delta v / v_{*} \approx 0.5$, the upper kHz-QPO being $v_{2}^{\mathrm{f}}=$ 

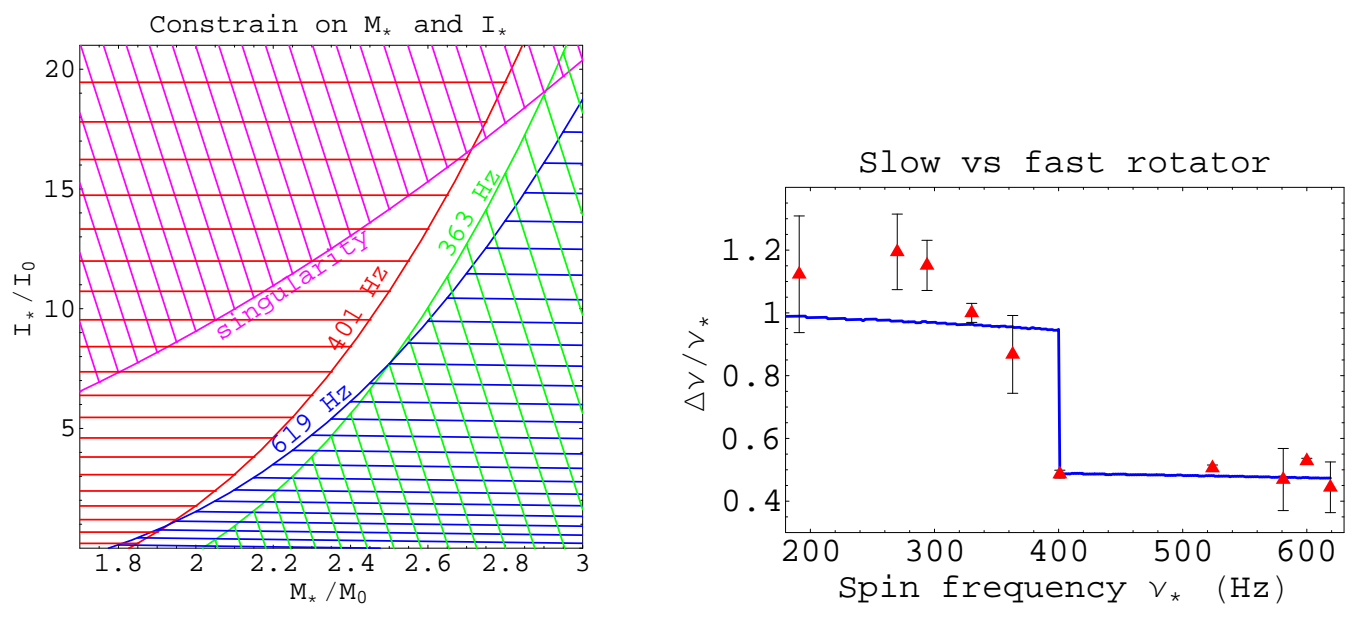

Figure 1: On the left, the four constraints in the $\left(M_{*}, I_{*}\right)$-plane, labeled with the spin frequency or marked by singularity. The hashed regions are forbidden. The minimum allowed mass and moment of inertia are around $M \approx 1.93 M_{\odot}$ and $I_{*} \approx 0.98 I_{0}$. On the right, observations of slow and fast rotators (red triangles) and fit obtained by our model (blue solid line). The best fit parameters are $M \approx 2.0-2.2 M_{\odot}$ and $I_{*} \approx 1-3 I_{0}$.

$2 v_{*}=1238 \mathrm{~Hz}$ and the lower kHz-QPO being $v_{1}^{\mathrm{f}}=1.5 v_{*}=929 \mathrm{~Hz}$. We conclude that for this particular system

$$
v_{\text {isco }}(619 \mathrm{~Hz}) \geq 1238 \mathrm{~Hz} .
$$

The last and general constrain is that there is no naked singularity in the Kerr metric or stated mathematically, $|\tilde{a}| \leq 1$. In terms of the moment of inertia, it means that

$$
I_{*} \leq \frac{G M_{*}^{2}}{2 \pi c v_{*}} .
$$

The less favorable case (most restrictive one) corresponds to $v_{*}=619 \mathrm{~Hz}$. This leads to

$$
\left(\frac{I_{*}}{10^{38} \mathrm{~kg} \mathrm{~m}^{2}}\right) \leq 2.26\left(\frac{M_{*}}{M_{\odot}}\right)^{2} .
$$

For later use, we introduce $I_{0}=10^{38} \mathrm{~kg} \mathrm{~m}^{2}$. All these constraints, Eq. (3.5)-(3.8), are summarized and shown in a $\left(M_{*}, I_{*}\right)$-plane depicted in Fig. 1, left part. The hashed regions are forbidden and only a small area in white survives around the first diagonal in the figure. This plot clearly emphasizes the existence of a lower and upper bound for both the mass and moment of inertia. We found the minimum values to be $M_{* \min }=1.9 M_{\odot}$ and $I_{* \min }=0.98 I_{0}$ whereas the maximum ones are $M_{* \max }=2.9 M_{\odot}$ and $I_{* \max }=19.1 I_{0}$. Neutron star structure models predict $I_{*}$ close to or slightly above $I_{0}$ so that we will favor the lower bounds and expect masses in the vicinity of $1.9 M_{\odot}$.

Indeed, the best fit parameters are $M \approx 2.0-2.2 M_{\odot}$ and $I_{*} \approx 0.5-1.5(10 \mathrm{~km})^{2} M_{\odot}$, leading to the right part of Fig. 1 where the spin rate is plotted on the $\mathrm{x}$-axis and the twin kHz-QPOs difference normalized to the spin rate is plotted on the y-axis. First, we retrieve the segregation between slow and fast rotators at the correct frequency as expected. Next, for fast spinning stars, the theoretical curve agrees very well with observations. Nevertheless, for slow rotation rates, the 
spread around unity is significant and cannot be explained in a straightforward way by our predictions. Clearly, some refinement of the model is still needed and under investigations, including other aspects of the plasma flow around an accreting magnetized neutron star.

\section{Discussion and Conclusion}

We investigated further the consequences of forced oscillations induced in an accretion disk to explain the twin kHz-QPOs in LMXBs. Our model is able to discriminate between slow and fast rotator as already shown in [3]. Moreover, with help on new data from a dozen rotators, we were able to constrain the average mass and moment of inertia of neutron stars. We found for the best fit $M \approx 2.0-2.2 M_{\odot}$ and $I_{*} \approx 0.5-1.5(10 \mathrm{~km})^{2} M_{\odot}$. Whereas the moment of inertia gives roughly the same value as those obtained from independent ways by solving the stellar structure, the neutron star mass appears rather large. This effect could be an artefact of its constancy from one binary system to another. Better fits suggests to look at each system individually. But this requires a much more detailed separate analysis of each binary with their own specificities (accretion rate, magnetic field strength for instance) and better observations. New time analyzing instruments like the HTRS (High Time Resolution Spectrometer) and spectroscopy projects on board IXO will give more insights into supra-nuclear matter and strong gravity physics [8].

\section{References}

[1] D. Page and S. Reddy. Dense Matter in Compact Stars: Theoretical Developments and Observational Constraints. Annual Review of Nuclear and Particle Science, 56:327-374, November 2006.

[2] J. M. Lattimer. Equation of state constraints from neutron stars. Ap\&SS, 308:371-379, April 2007.

[3] J. Pétri. An explanation for the kHz-QPO twin peaks separation in slow and fast rotators. A\&A, 439:L27-L30, August 2005.

[4] H. X. Yin, C. M. Zhang, Y. H. Zhao, Y. J. Lei, J. L. Qu, L. M. Song, and F. Zhang. The correlations between the spin frequencies and kHz QPOs of neutron stars in LMXBs. A\&A, 471:381-384, August 2007.

[5] J. Pétri. Constraining the mass and moment of inertia of neutron stars from quasi-periodic oscillations in X-ray binaries. Ap\&SS, 331:555-563, February 2011.

[6] M. C. Miller, F. K. Lamb, and D. Psaltis. Sonic-Point Model of Kilohertz Quasi-periodic Brightness Oscillations in Low-Mass X-Ray Binaries. ApJ, 508:791-830, December 1998.

[7] M. Méndez and T. Belloni. Is there a link between the neutron-star spin and the frequency of the kilohertz quasi-periodic oscillations? MNRAS, 381:790-796, October 2007.

[8] D. Barret, T. Belloni, S. Bhattacharyya, M. Gilfanov, E. Gogus, J. Homan, M. Méndez, J. M. Miller, M. C. Miller, S. Mereghetti, S. Paltani, J. Poutanen, J. Wilms, and A. A. Zdziarski. Science with the XEUS high time resolution spectrometer. In Society of Photo-Optical Instrumentation Engineers (SPIE) Conference Series, volume 7011 of Society of Photo-Optical Instrumentation Engineers (SPIE) Conference Series, August 2008. 